\title{
Influence of Concentration Distribution on Cross Diffusion Level in Building Room
}

\author{
Du Zhehua $^{1}$ \\ ${ }^{1}$ Wuhan Second Ship Design and Research Institute, 430205 Wuhan, China
}

\begin{abstract}
The relationship of contaminant gas concentration distribution influence on cross diffusion character and level among temperature, humidity and contaminant gas concentration was obtained according to the non-equilibrium thermodynamic theory. The cross diffusion character and level under different contaminant gas concentration distribution were discussed, combining real temperature and humidity in building room. The results show that temperature grads and vapor mass grads are less than zero when contaminant gas mass grads and additional diffusion coefficient are both positive or negative, otherwise the two grads are more than zero. And the higher the initial temperature and humidity levels, the greater the absolute values of temperature grads and vapor mass grads, with the same contaminant gas mass grads and additional diffusion coefficient. While the influence of initial temperature level is finite, and that of initial humidity level is remarkable.
\end{abstract}

\section{Introduction}

Gas pollutants are affected by multi-physical fields (temperature field, humidity field and self-concentration field) in the process of diffusion and transmission. Its diffusion and migration characteristics are not only related to its own concentration distribution, but also affected by the distribution characteristics of temperature and humidity[1-3]. The reason for this effect is that the coexistence of multiple physical fields not only makes gas components self-diffusion, but also is affected by cross-diffusion[4-5].Therefore, the correct understanding of the characteristics and level of cross diffusion of indoor gas pollutants is one of the prerequisites for effective control of gas pollutants. Based on irreversible thermodynamic analysis [6], this paper focuses on the influence of pollution gas concentration distribution characteristics on the cross-diffusion level.

\section{Cross diffusion transfer relationship}

For an irreversible process in a non-equilibrium thermodynamic system, there is the following relationship between thermodynamic flow and thermodynamic force, as shown in equation (1).

$$
J_{i}=\sum_{j}\left(\frac{\delta J_{i}}{\delta X_{j}}\right) X_{j}+\frac{1}{2} \sum_{j, k}\left(\frac{\delta^{2} J_{i}}{\delta X_{j} \delta X_{k}}\right) X_{j} X_{k}+\cdots
$$

In the linear non-equilibrium region, ignoring the higher-order terms can get equation (2).

$$
J_{i}=\sum_{j=1}^{n} L_{i j} X_{j}
$$

In the equation, phenomenological coefficient

$$
L_{i j}=\left(\frac{\delta J_{i}}{\delta X_{j}}\right)_{0}
$$

According to the transformation relation of $n$ components to $(n-1)$ components, the corresponding thermodynamic force is written in the form of Equations (4) and (5).

$$
\begin{gathered}
X_{q}=-\nabla\left(\frac{1}{T}\right) \\
X_{i}=-\sum_{i=1}^{n-1} A_{i j} \nabla\left(\frac{\mu_{j}}{T}\right) \\
A_{k j}=\delta_{k j}+\frac{\varphi_{j}}{\varphi_{n}}, i, j, k=1,2, \cdots, n-1 \\
T \text {-- Thermodynamic temperature } \\
\mu_{j}-\text { - Chemical potential } \\
\varphi_{j} \text {-- Mass percentage of } j \text { component }
\end{gathered}
$$

Therefore, the corresponding entropy production rate can be expressed as equation (7).

$$
\sigma=-J_{q} \nabla\left(\frac{1}{T}\right)-\sum_{i=1}^{n-1} A_{i j} \nabla\left(\frac{\mu_{j}}{T}\right)
$$

Thus the corresponding linear phenomenological equations are equations (8) and (9).

$$
\begin{aligned}
& J_{q}=L_{t t} \nabla\left(\frac{1}{T}\right)+\sum_{j, k=1}^{n-1} L_{t k}\left[-A_{k j} \nabla\left(\frac{\mu_{j}}{T}\right)\right] \\
& J_{i}=L_{i t} \nabla\left(\frac{1}{T}\right)+\sum_{j, k=1}^{n-1} L_{i k}\left[-A_{k j} \nabla\left(\frac{\mu_{j}}{T}\right)\right]
\end{aligned}
$$

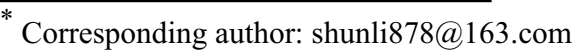


The corresponding Onsager reciprocal relations in equations (8) and (9) are equations (10) and (11).

$$
\begin{aligned}
& L_{i t}=L_{t i} \\
& L_{i k}=L_{k i}
\end{aligned}
$$

According to Curie's theorem, there are cross-effects in the corresponding thermodynamic forces in the diffusion and transfer process of building space heat, water vapor components, and polluting gas components. Without considering other thermodynamic forces, the corresponding diffusion flows can be obtained as Equations (12), (13) and (14) respectively.

$$
\begin{aligned}
& J_{q}=L_{c t} X_{c}+L_{h t} X_{h}+L_{t t} X_{t} \\
& J_{h}=L_{c h} X_{c}+L_{h h} X_{h}+L_{h t} X_{t} \\
& J_{c}=L_{c c} X_{c}+L_{c h} X_{h}+L_{c t} X_{t}
\end{aligned}
$$

According to equations (4) and (5), the forms of each thermodynamic force in equations (12), (13) and (14) can be obtained as equations (15), (16) and (17).

$$
\begin{gathered}
X_{t}=-\frac{1}{T^{2}} \nabla T \\
X_{h}=-\left[\frac{A_{h h}}{T}\left(\frac{\partial \mu_{h}}{\partial c}\right)_{p, T, h}+\frac{A_{h c}}{T}\left(\frac{\partial \mu_{c}}{\partial c}\right)_{p, T, h}\right] \nabla c \\
-\left[\frac{A_{h h}}{T}\left(\frac{\partial \mu_{h}}{\partial h}\right)_{p, T, c}+\frac{A_{h c}}{T}\left(\frac{\partial \mu_{c}}{\partial h}\right)_{p, T, c}\right] \nabla h \\
X_{c}=-\left[\frac{A_{c c}}{T}\left(\frac{\partial \mu_{c}}{\partial h}\right)_{p, T, c}+\frac{A_{c h}}{T}\left(\frac{\partial \mu_{h}}{\partial h}\right)_{p, T, c}\right] \nabla h \\
-\left[\frac{A_{c c}}{T}\left(\frac{\partial \mu_{c}}{\partial c}\right)_{p, T, h}+\frac{A_{c h}}{T}\left(\frac{\partial \mu_{h}}{\partial c}\right)_{p, T, h}\right] \nabla c
\end{gathered}
$$

$h, c$-- Mass fraction of water vapor component and pollutant gas component.

In order to replace the phenomenological coefficients in equations (12), (13) and (14), the following coefficients are introduced in equations (18) - (26).

$$
\left.\begin{array}{c}
\lambda=\frac{L_{t t}}{T^{2}} \\
D_{t h}=\frac{L_{h t}}{\rho(1-c-h) h T^{2}} \\
D_{t c}=\frac{L_{c t}}{\rho(1-c-h) c T^{2}} \\
D_{c c}=\frac{1}{\rho(1-c-h) T}\left\{\begin{array}{l}
{\left[L_{c h}(1-h)+L_{h h} c\right]\left(\frac{\partial \mu_{c}}{\partial h}\right)_{p, T, c}} \\
+\left[L_{c h} h+L_{h h}(1-c)\right]\left(\frac{\partial \mu_{h}}{\partial h}\right)_{p, T, c}
\end{array}\right\} \\
{\left[L_{c c}(1-h)+L_{c h} c\right]\left(\frac{\partial \mu_{c}}{\partial c}\right)_{p, T, h}} \\
+\left[L_{c c} h+L_{c h}(1-c)\right]\left(\frac{\partial \mu_{h}}{\partial c}\right)_{p, T, h}
\end{array}\right\}
$$

$$
\begin{aligned}
& D_{h t}=\frac{1}{\rho(1-c-h) c T^{2}}\left\{\begin{array}{l}
{\left[L_{c t}(1-h)+L_{h t} c\right]\left(\frac{\partial \mu_{c}}{\partial h}\right)_{p, T, c}} \\
+\left[L_{c t} h+L_{h t}(1-c)\right]\left(\frac{\partial \mu_{h}}{\partial h}\right)_{p, T, c}
\end{array}\right\} \\
& D_{c t}=\frac{1}{\rho(1-c-h) h T^{2}}\left\{\begin{array}{l}
{\left[L_{c t}(1-h)+L_{h t} c\right]\left(\frac{\partial \mu_{c}}{\partial c}\right)_{p, T, h}} \\
+\left[L_{c t} h+L_{h t}(1-c)\right]\left(\frac{\partial \mu_{h}}{\partial c}\right)_{p, T, h}
\end{array}\right\} \\
& D_{c h}=\frac{1}{\rho(1-c-h) h T^{2}}\left\{\begin{array}{l}
{\left[L_{c h}(1-h)+L_{h h} c\right]\left(\frac{\partial \mu_{c}}{\partial c}\right)_{p, T, h}} \\
+\left[L_{c h} h+L_{h h}(1-c)\right]\left(\frac{\partial \mu_{h}}{\partial c}\right)_{p, T, h}
\end{array}\right\} \\
& D_{h c}=\frac{1}{\rho(1-c-h) c T^{2}}\left\{\begin{array}{l}
{\left[L_{c c}(1-h)+L_{c h} c\right]\left(\frac{\partial \mu_{c}}{\partial h}\right)_{p, T, c}} \\
+\left[L_{c c} h+L_{c h}(1-c)\right]\left(\frac{\partial \mu_{h}}{\partial h}\right)_{p, T, c}
\end{array}\right\}
\end{aligned}
$$

Substitute the above coefficients into equations (12), (13) and (14), equations (27), (28) and (29) can be obtained.

$$
\begin{aligned}
& J_{q}=-\rho h T D_{c t} \nabla c-\rho c T D_{h t} \nabla h-\lambda \nabla T \\
& J_{h}=-\rho h T D_{c h} \nabla c-\rho D_{h h} \nabla h-\rho(1-c-h) h D_{t h} \nabla T \\
& J_{c}=-\rho D_{c c} \nabla c-\rho c T D_{h c} \nabla h-\rho(1-c-h) c D_{t c} \nabla T
\end{aligned}
$$

Therefore, the transfer relationship including the cross-diffusion effect in the process of building space transfer is established.

\section{Influence of concentration distribution on the level of cross diffusion}

The distribution characteristics of indoor pollutant gas concentration have two effects on the cross-diffusion transfer process. On the one hand, the diffusion additional thermal effect is caused by the concentration gradient of pollutant gas. A temperature gradient is formed under the action of diffusion plus thermal effect. The diffusion heat flow in the opposite direction to the diffusion additional heat effect is produced by temperature gradient. On the other hand, the concentration gradient of pollutant gas will also cause water vapor component to produce diffusion effect. Humidity gradient is formed under the action of diffusion effect of diffusion additive. The humidity gradient produces mass diffusion in the opposite direction to the diffusion effect. When the diffused heat effect reaches equilibrium with the diffused heat flow in the opposite direction and the diffused additional mass diffusion effect reaches equilibrium with the mass diffusion in the opposite direction, the whole thermodynamic system is in a stationary state. Therefore, in order to analyze the influence of room pollution gas concentration distribution characteristics, cross diffusion coefficient is introduced, as shown in equations (30), (31), (32) and (33). 


$$
\begin{gathered}
D u_{h t}=\frac{D_{h t}}{\lambda} \\
D u_{c t}=\frac{D_{c t}}{\lambda} \\
G_{c h}=\frac{D_{c h}}{D_{h h}} \\
S_{t h}=\frac{D_{t h}}{D_{h h}}
\end{gathered}
$$

Meanwhile, according to the steady-state characteristics, the following equations are obtained.

$$
\begin{gathered}
J_{q}=0 \\
J_{h}=0
\end{gathered}
$$

Thus, from equations (27) and (29), equations (36) and (37) can be obtained.

$$
\begin{aligned}
& \rho h T D u_{c t} \nabla c+\rho c T D u_{h t} \nabla h+\nabla T=0 \\
& h T G_{c h} \nabla c+\nabla h+(1-c-h) h S_{t h} \nabla T=0
\end{aligned}
$$

Since $\mathrm{T}$ and $\mathrm{H}$ in equations (36) and (37) are formed based on $\mathrm{C}$, the second term on the left in equation (36) and the third term on the left in equation (37) are small quantities compared with other terms and can be approximately ignored. Thus we can get equation (38) and equation (39).

$$
\begin{gathered}
\nabla T=-\rho h T D u_{c t} \nabla c \\
\nabla h=-h T G_{c h} \nabla c
\end{gathered}
$$

Based on the actual fluctuation range of indoor temperature and relative humidity, the initial indoor temperature level and humidity level are determined in accordance with 6 scenarios, as shown in Table 1 .

Table 1. Initial temperature and relative humidity level

\begin{tabular}{|c|c|c|}
\hline situation & Temperature $/{ }^{\circ} \mathbf{C}$ & Humidity $/ \%$ \\
\hline 1 & 20 & 60 \\
\hline 2 & 26 & 60 \\
\hline 3 & 30 & 60 \\
\hline 4 & 26 & 40 \\
\hline 5 & 26 & 60 \\
\hline 6 & 26 & 80 \\
\hline
\end{tabular}

Figure 1 and Figure 2 respectively show the influence of the pollution gas concentration distribution characteristics on temperature distribution and water vapor composition distribution under the effect of cross diffusion for six situations listed in Table 1.
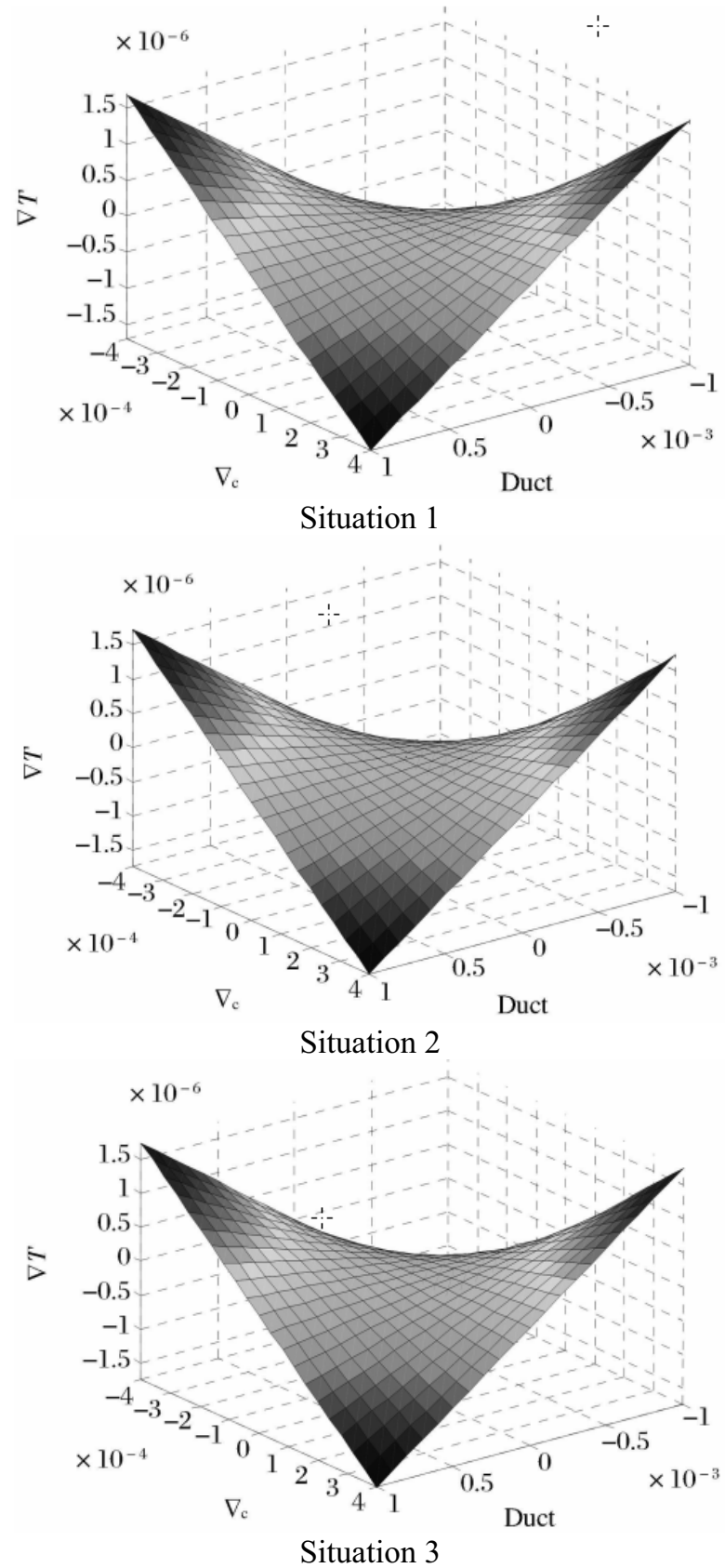

Fig. 1. Influence of temperature distribution

As can be seen from Figure 1, when $\nabla c$ is less than zero, if the $D u_{c t}$ is greater than zero, then the additional diffusion heat flow direction is opposite to $\nabla c$, and the temperature gradient $\nabla T$ is greater than zero. If $D u_{c t}$ is less than zero, the additional diffusion heat flow direction is the same as $\nabla c$, then the resulting temperature gradient $\nabla T$ is less than zero. For the case that $\nabla c$ is greater than zero, combined with the positive and negative of Duct, it can be inferred that the sign of $\nabla T$ is opposite to the result when $\mathrm{c}$ is less than zero. At the same time, $\nabla T$ formed by the additional thermal effect of diffusion will produce self-diffusion, which is opposite to the direction of additional thermal diffusion. When the process time reaches characteristic time, selfdiffusion and diffusion-additional thermal diffusion 
reach equilibrium, and the system behaves in a steady state.

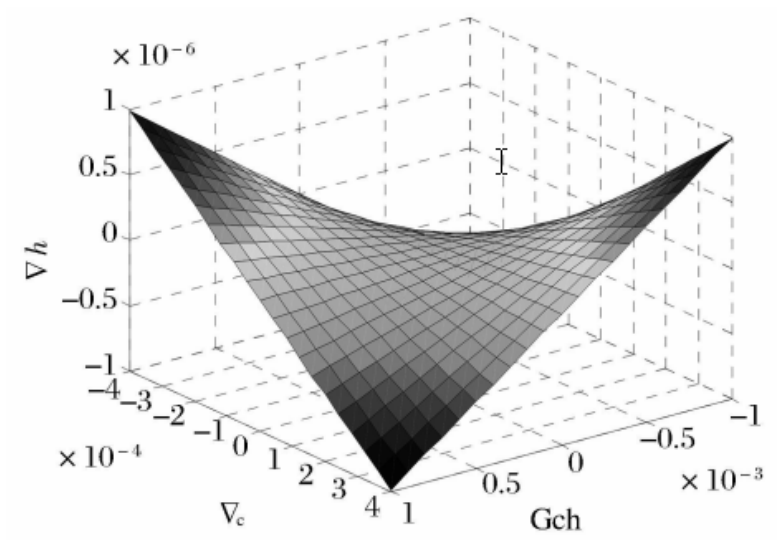

Situation 4

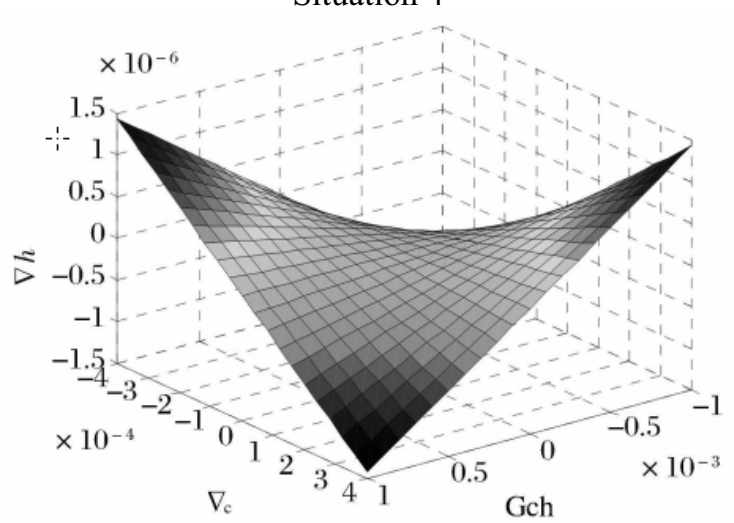

Situation 5

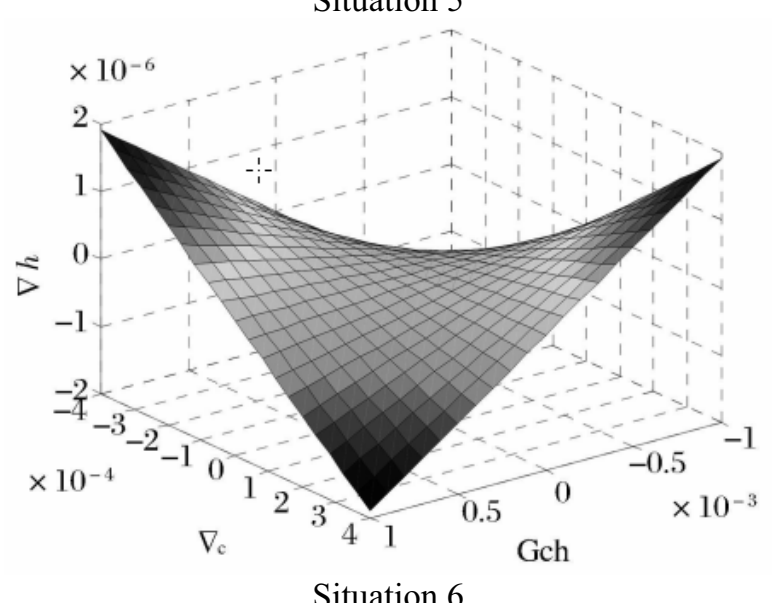

Situation 6

Fig. 2. Influence of water vapor composition

It can be found from Figure 2 that when $\nabla c$ is greater than zero, if $G_{c h}$ is greater than zero, the direction of the diffusion flow of water vapor components is opposite to $\nabla c$, and the resulting $\nabla h$ is less than zero. If $G_{c h}$ is less than zero, and the direction of the diffusion flow of water vapor components is the same as c, the resulting $\nabla h$ is greater than zero. For the case where $\nabla c$ is less than zero, combining the sign of $G_{c h}$, it can be inferred that the sign of $\nabla h$ is opposite to the result when $\nabla c$ is greater than zero. Moreover, $\nabla h$ formed by the diffusion effect of diffusive additive will produce self-diffusion, which is opposite to the diffusion direction of diffusive additive. When the process time reaches characteristic time, self-diffusion and diffusionadditional thermal diffusion reach equilibrium, and the system behaves in a steady state.

\section{Conclusion}

Based on irreversible thermodynamic analysis, the relationship between the distribution characteristics of indoor pollutant gas concentration in buildings and the cross-diffusion level and characteristics is established.

When the pollutant gas composition gradient and the diffusion additional diffusion coefficient have the same sign, the temperature gradient and the water vapor composition gradient formed are less than zero, otherwise the temperature gradient and the water vapor composition gradient formed are greater than zero.

Under the same pollutant gas composition gradient and the additional thermal effect of diffusion, the higher the initial indoor temperature level, the greater the absolute value of the temperature gradient formed, but the influence of the initial temperature level is limited.

Under the same pollutant gas composition gradient and the diffusion effect of diffusive additives, the higher the initial indoor humidity level, the greater the absolute value of water vapor composition gradient, and the influence of the initial humidity level is significant.

\section{References}

1. B.F. $\mathrm{Yu}, \mathrm{Z} . \mathrm{B}$. $\mathrm{Hu}$, International Journal of Refrigeration, 32, 3 (2009)

2. M. Stranger, Indoor Air, 18, 454 (2018)

3. W. Peder, Atmospheric Environment, 32, 2659 (1998)

4. L.J. Kempers, Journal of Chemical Physics, 115, 6342 (2001)

5. O. Lhost, J.K. Platten, Physical Review A, 40, 6415 (1989)

6. I. Ilya, Microgravity Sci. Technol., 10, 1007 (2008) 